\title{
Study of baryon octet charge form factors in perturbative chiral quark model
}

\author{
X. Y. Liu*, K. Khosonthongkee, A. Limphirat and Y. Yan ${ }^{\dagger}$ \\ School of Physics, Institute of Science, Suranaree University of Technology \\ Nakhon Ratchasima 30000, Thailand \\ Thailand Center of Excellence in Physics (ThEP) \\ Commission on Higher Education, Bangkok 10400, Thailand \\ *lxy_gzu2005@126.com \\ †yupeng@sut.ac.th
}

Published 16 April 2014

\begin{abstract}
The charge form factors of baryon octet are studied in the perturbative chiral quark model (PCQM). The relativistic quark wave function is extracted by fitting the theoretical results of the nucleon charge form factors to the experimental data and the predetermined quark wave function is applied to study the charge form factors of other octet baryons. The PCQM results are found, based on the predetermined quark wave function, in good agreement with the experimental data.
\end{abstract}

Keywords: Charge form factor; octet baryon; PCQM.

PACS Numbers: 14.20.-c, 13.40.Gp, 12.39.-x

\section{Introduction}

The perturbative chiral quark model (PCQM) was developed a decade ago ${ }^{1,2}$ and has been successfully applied to the electromagnetic and axial form factors of baryons, ${ }^{3-6}$ low-energy meson-baryon scatterings, ${ }^{7}$ electromagnetic excitations of nucleon resonances, ${ }^{8}$ nucleon polarizabilities, ${ }^{9}$ neutron electric dipole form factor, ${ }^{10}$ etc. These studies demonstrate the PCQM is one of the effective models in the low energy hadron physics, and indicate that the virtual meson cloud exists mainly outside of the quark core. But the PCQM theoretical results ${ }^{3-6}$ of the electromagnetic and axial form factors of baryons are in good agreement with experimental data only at very low momentum transfer, descending quickly with the momentum transfer increasing. In those works, ${ }^{3-6}$ a variational Gaussian ansatz have been employed for the quark wave function. One may argue that it is the Gaussian-type quark

This is an Open Access article published by World Scientific Publishing Company. It is distributed under the terms of the Creative Commons Attribution 3.0 (CC-BY) License. Further distribution of this work is permitted, provided the original work is properly cited. 
wave function of baryons which leads to the theoretical predictions for the form factors of baryons consistent well with experimental data only at very low momentum transfer. In this work we extract the quark wave function by fitting the PCQM theoretical result of the nucleon charge form factors to the experimental data, and study the charge form factors of other octet baryons with the predetermined wave function.

The paper is organized as follows. In Section 2 we introduce the PCQM and present the theoretical expressions of octet baryon charge form factors in the framework of the PCQM. The extracted quark wave function and numerical results are given in Section 3.

\section{Charge form Factors in the PCQM}

The PCQM ${ }^{1,2}$ is based on an effective chiral Lagrangian describing baryons by a core of the three valence quarks, moving in a central Dirac field with $V_{\text {eff }}(r)=$ $S(r)+\gamma_{0} V(r)$, where $r=|\vec{x}|$. In order to respect chiral symmetry, a cloud of Goldstone bosons $(\pi, K$ and $\eta)$ is included as small fluctuations around the threequark core in SU(3) extension. With an unitary chiral rotation, as shown in Refs. 5, 7, the Weinberg-type Lagrangian of the PCQM is derived,

$$
\begin{aligned}
\mathcal{L}^{W}(x) & =\mathcal{L}_{0}(x)+\mathcal{L}_{I}^{W}(x)+o(\vec{\pi}), \\
\mathcal{L}_{0}(x) & =\bar{\psi}(x)\left[i \not \partial-\gamma^{0} V(r)-S(r)\right] \psi(x)-\frac{1}{2} \Phi_{i}(x)\left(\square+M_{\Phi}^{2}\right) \Phi^{i}(x), \\
\mathcal{L}_{I}^{W}(x) & =\frac{1}{2 F} \partial_{\mu} \Phi_{i}(x) \bar{\psi}(x) \gamma^{\mu} \gamma^{5} \lambda^{i} \psi(x)+\frac{f_{i j k}}{4 F^{2}} \Phi_{i}(x) \partial_{\mu} \Phi_{j}(x) \bar{\psi}(x) \gamma^{\mu} \lambda_{k} \psi(x),
\end{aligned}
$$

where $f_{i j k}$ are the totally antisymmetric structure constant of $S U(3)$, the pion decay constant $F=88 \mathrm{MeV}$ in the chiral limit, $\Phi_{i}$ are the octet meson fields, and $\psi(x)$ is the triplet of the $u, d$, and $s$ quark fields taking the form

$$
\psi(x)=\left(\begin{array}{l}
u(x) \\
d(x) \\
s(x)
\end{array}\right) .
$$

The quark field $\psi(x)$ could be expanded in

$$
\psi(x)=\sum_{\alpha}\left(b_{\alpha} u_{\alpha}(\vec{x}) e^{-i \mathcal{E}_{\alpha} t}+d_{\alpha}^{\dagger} v_{\alpha}(\vec{x}) e^{i \mathcal{E}_{\alpha} t}\right)
$$

where $b_{\alpha}$ and $d_{\alpha}^{\dagger}$ are the single quark annihilation and antiquark creation operators. The ground state quark wave function $u_{0}(\vec{x})$ may, in general, be expressed as

$$
u_{0}(\vec{x})=\left(\begin{array}{c}
g(r) \\
i \vec{\sigma} \cdot \hat{x} f(r)
\end{array}\right) \chi_{s} \chi_{f} \chi_{c}
$$

where $\chi_{s}, \chi_{f}$ and $\chi_{c}$ are the spin, flavor and color quark wave functions, respectively. 
The calculation technique in the PCQM is based on the Gell-Mann and Low theorem, ${ }^{11}$ in which the expectation value of an operator $\hat{O}$ can be calculated from

$$
\langle\hat{O}\rangle={ }^{B}\left\langle\phi_{0}\left|\sum_{n=0}^{\infty} \frac{i^{n}}{n !} \int d^{4} x_{1} \cdots \int d^{4} x_{n} T\left[\mathcal{L}_{I}^{W}\left(x_{1}\right) \cdots \mathcal{L}_{I}^{W}\left(x_{n}\right) \hat{O}\right]\right| \phi_{0}\right\rangle_{c}^{B},
$$

where the state vector $\left|\phi_{0}\right\rangle^{B}$ corresponds to the unperturbed three-quark states projected onto the respective baryon states, which are constructed in the framework of the $S U(6)$ spin-flavor and $S U(3)$ color symmetry. The subscript $c$ in Eq. (7) refers to contributions from connected graphs only. $\mathcal{L}_{I}^{W}(x)$ is the quark-meson interaction Lagrangian as given in Eq. (3). In the framework of the PCQM, the charge form factors of octet baryons in the Breit frame are defined by

$$
\begin{aligned}
\chi_{B_{s}^{\prime}}^{\dagger} \chi_{B_{s}} G_{E}^{B}\left(Q^{2}\right)= & { }^{B}\left\langle\phi_{0}\right| \sum_{n=0}^{n} \frac{i^{n}}{n !} \int \delta(t) d^{4} x d^{4} x_{1} \cdots d^{4} x_{n} e^{-i q \cdot x} \\
& \times T\left[\mathcal{L}_{I}^{W}\left(x_{1}\right) \cdots \mathcal{L}_{I}^{W}\left(x_{n}\right) j^{0}(x)\right]\left|\phi_{0}\right\rangle_{c}^{B},
\end{aligned}
$$

Here, $G_{E}^{B}\left(Q^{2}\right)$ are the charge form factors of octet baryons with the space-like squared momentum transfer $Q^{2}$, which is carried out by the electromagnetic current. In the Breit frame, the initial momentum of the baryons is $p=(E,-\vec{q} / 2)$, the final momentum is $p=(E, \vec{q} / 2)$, and the four-momentum of the photon is $q=(0, \vec{q})$. Thus, $Q^{2}=-q^{2}=\vec{q}^{2} \cdot \chi_{B_{s}}$ and $\chi_{B_{s^{\prime}}}^{\dagger}$ are the baryon spin wave functions in the initial and final states, and $j^{\mu}$ is the electromagnetic current

$$
j^{\mu}=j_{\psi}^{\mu}+j_{\Phi}^{\mu}+j_{\psi \Phi}^{\mu}+\delta j_{\psi}^{\mu}
$$

which contains the quark current $j_{\psi}^{\mu}$, the charged pseudoscalar mesons current $j_{\Phi}^{\mu}$, the quark-meson coupling current $j_{\psi \Phi}^{\mu}$, and $\delta j_{\psi}^{\mu}$, a current arising from the counterterm. The currents in the above equation take the forms,

$$
\begin{aligned}
j_{\psi}^{\mu} & =\bar{\psi} \gamma^{\mu} \mathcal{Q} \psi \\
j_{\Phi}^{\mu} & =\left[f_{3 i j}+\frac{f_{8 i j}}{\sqrt{3}}\right] \Phi_{i} \partial^{\mu} \Phi_{j} \\
j_{\psi \Phi}^{\mu} & =\left[f_{3 i j}+\frac{f_{8 i j}}{\sqrt{3}}\right] \frac{\Phi_{j}}{2 F} \bar{\psi} \gamma^{\mu} \gamma^{5} \lambda_{i} \psi, \\
\delta j_{\psi}^{\mu} & =\bar{\psi}(Z-1) \gamma^{\mu} \mathcal{Q} \psi,
\end{aligned}
$$

where $\mathcal{Q}$ is the quark charge matrix $\mathcal{Q}=\operatorname{diag}\{2 / 3,-1 / 3,-1 / 3\}$, and the renormalization constants $\hat{Z}$ and $\hat{Z}_{s}$ are defined as

$$
\hat{Z}=1-\frac{3}{4(2 \pi F)^{2}} \int_{0}^{\infty} d k k^{4} F_{I}^{2}\left(k^{2}\right)\left[\frac{1}{\omega_{\pi}^{3}\left(k^{2}\right)}+\frac{2}{3 \omega_{K}^{3}\left(k^{2}\right)}+\frac{1}{9 \omega_{\eta}^{3}\left(k^{2}\right)}\right]
$$




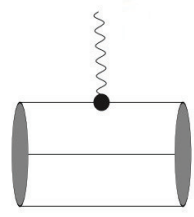

(a)

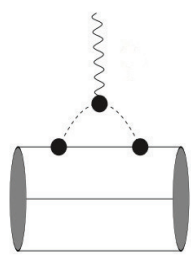

(c)

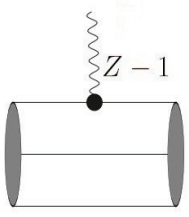

(b)

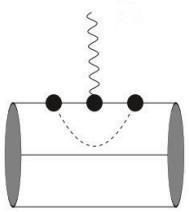

(d)

Fig. 1. Diagrams contributing to the charge form factors of the baryon octet: three-quark diagram (a), three-quark counterterm diagram (b), meson cloud diagram (c), and vertex correction diagram $(d)$.

$$
\hat{Z}_{s}=1-\frac{1}{(2 \pi F)^{2}} \int_{0}^{\infty} d k k^{4} F_{I}^{2}\left(k^{2}\right)\left[\frac{1}{\omega_{K}^{3}\left(k^{2}\right)}+\frac{1}{3 \omega_{\eta}^{3}\left(k^{2}\right)}\right],
$$

with $\omega_{\Phi}\left(k^{2}\right)=\sqrt{M_{\Phi}^{2}+k^{2}}$ and the vertex function $F_{I}(k)$ for the $q q \Phi$ system taking the form

$$
F_{I}(k)=2 \pi \int_{0}^{\infty} d r \int_{0}^{\pi} d \theta r^{2} \sin \theta e^{i k r \cos \theta}\left[g(r)^{2}+f(r)^{2} \cos 2 \theta\right] .
$$

In accordance with the interaction Lagrangian $\mathcal{L}_{I}^{W}(x)$ in Eq. (3) and the electromagnetic current $j^{\mu}$ in Eqs. (10)-(13), there are four Feynman diagrams, as shown in Eq. (1), contributing to the charge form factors to the one-loop order. ${ }^{3,4}$ The contributions of these diagrams are derived as follows:

(a) Three-quark core leading-order diagram (LO)

$$
\left.G_{E}^{B}\left(Q^{2}\right)\right|_{L O}=\left.a_{1}^{B} G_{E}^{p}\left(Q^{2}\right)\right|_{L O} ^{3 q}
$$

where

$$
\left.G_{E}^{p}\left(Q^{2}\right)\right|_{L O} ^{3 q}=2 \pi \int_{0}^{\infty} d r \int_{0}^{\pi} d \theta r^{2} \sin \theta\left[g(r)^{2}+f(r)^{2}\right] e^{i Q r \cos \theta}
$$

(b) Three-quark core counterterm $\operatorname{diagram}(\mathrm{CT})$

$$
\left.G_{E}^{B}\left(Q^{2}\right)\right|_{C T} ^{3 q}=\left.\left[a_{2}^{B}(\hat{Z}-1)+a_{3}^{B}\left(\hat{Z}_{s}-1\right)\right] G_{E}^{p}\left(Q^{2}\right)\right|_{L O} ^{3 q}
$$


(c) Meson-cloud diagram (MC)

$\left.G_{E}^{B}\left(Q^{2}\right)\right|_{M C}=\left.\frac{1}{2(2 \pi F)^{2}} \int_{0}^{\infty} d k \int_{-1}^{1} d x k^{2}\left(k^{2}+k Q x\right) F_{I}(k) F_{I}\left(k_{+}\right) t_{E}^{B}\left(k^{2}, Q^{2}, x\right)\right|_{M C}$,

where

$$
\begin{array}{r}
\left.t_{E}^{B}\left(k^{2}, Q^{2}, x\right)\right|_{M C}=a_{4}^{B} C_{\pi}\left(k^{2}, Q^{2}, x\right)+a_{5}^{B} C_{K}\left(k^{2}, Q^{2}, x\right), \\
C_{\Phi}\left(k^{2}, Q^{2}, x\right)=\frac{1}{\omega_{\Phi}\left(k^{2}\right) \omega_{\Phi}\left(k_{+}^{2}\right)\left[\omega_{\Phi}\left(k^{2}\right)+\omega_{\Phi}\left(k_{+}^{2}\right)\right]}, \\
k_{+}=\sqrt{k^{2}+Q^{2}+2 k \sqrt{Q^{2}} x}
\end{array}
$$

(d) Vertex-correction diagram (VC)

$$
\left.G_{E}^{B}\left(Q^{2}\right)\right|_{V C}=\left.\frac{1}{2(2 \pi F)^{2}} \int_{0}^{\infty} d k k^{4} F_{I}^{2}(k) G_{E}^{p}\left(Q^{2}\right)\right|_{L O} ^{3 q}\left[\frac{a_{6}^{B}}{\omega_{\pi}^{3}\left(k^{2}\right)}+\frac{a_{7}^{B}}{\omega_{K}^{3}\left(k^{2}\right)}+\frac{a_{8}^{B}}{\omega_{\eta}^{3}\left(k^{2}\right)}\right] \text {. }
$$

The constants $a_{i}^{B}$, which depend on the spin and flavor of baryons, have been listed in Ref. 4.

\section{Model Quark Wave Function and Numerical Results}

The theoretical expressions in the above section show that the charge form factors are mainly determined by the wave function of the quark core. In the previous works, ${ }^{3,4}$ the Gaussian-type quark wave function of baryons is employed and the theoretical results for the form factors of baryons are consistent well with experimental data at low momentum transfer $Q^{2}$. Instead of assuming a certain type, we extract in this work the quark wave function by adjusting our theoretical result of the nucleon charge form factors to the experimental data. The radial quark wave functions $g(r)$ and $f(r)$, the upper and lower components in the ground state, are expanded in the complete set of Sturmian functions $S_{n l}(r),{ }^{12}$

$$
\begin{aligned}
& g(r)=\sum_{n} A_{n} \frac{S_{n 0}(r)}{r}, \\
& f(r)=r \sum_{n} B_{n} \frac{S_{n 0}(r)}{r},
\end{aligned}
$$

with

$$
S_{n l}(r)=\left[\frac{n !}{(n+2 l+1) !}\right]^{\frac{1}{2}}(2 b r)^{l+1} e^{-b r} L_{n}^{2 l+1}(2 b r)
$$

where $L_{n}^{2 l+1}(x)$ are Laguerre polynomials, and $b$ is the length parameter of Sturmian functions. 
Table 1. The normalized model parameters determined by fitting the theoretical result of the nucleon charge form factor to the experimental data.

\begin{tabular}{rrr}
\hline$n$ & \multicolumn{1}{c}{$A_{n}^{\prime}$} & $B_{n}^{\prime}$ \\
\hline 0 & 0.21995228 & 0.13878775 \\
1 & -0.00776088 & 0.02891815 \\
2 & 0.00092791 & 0.01014163 \\
3 & -0.01348490 & 0.00065646 \\
4 & -0.00591343 & -0.00048920 \\
\hline
\end{tabular}

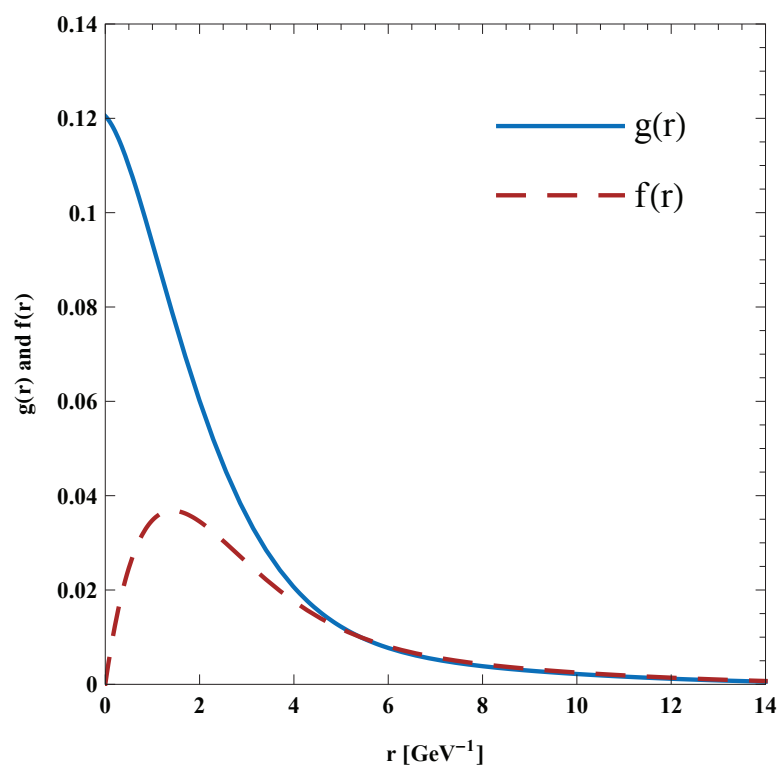

Fig. 2. Normalized radial wave functions of the valence quarks for the upper component $g(r)$ (solid line) and the lower component $f(r)$ (dashed line).

It is found that a basis of five Sturmian functions $(n=0,1,2,3,4)$ is good enough to let our theoretical result of the proton charge form factor fit to experimental data. The Sturmian function length parameter is fixed to be $b=0.5 \mathrm{GeV}$, and the expansion coefficients $A_{n}$ and $B_{n}$ are compiled in Table 1 , where we redefine $A_{n}^{\prime}=A_{n} b^{-1 / 2}$ and $B_{n}^{\prime}=B_{n} b^{-3 / 2}$ to let the $A_{n}^{\prime}$ and $B_{n}^{\prime}$ be dimensionless. Shown in Fig. 2 is the quark radial wave functions $g(r)$ and $f(r)$. Note that in the calculation the quark wave function is normalized according to $\int d^{3} \vec{x} u^{\dagger}(\vec{x}) u(\vec{x})=1$. Larger bases have been applied, but the fitted results of the quark wave functions appear the same as the one with the basis of five Sturmian functions. 

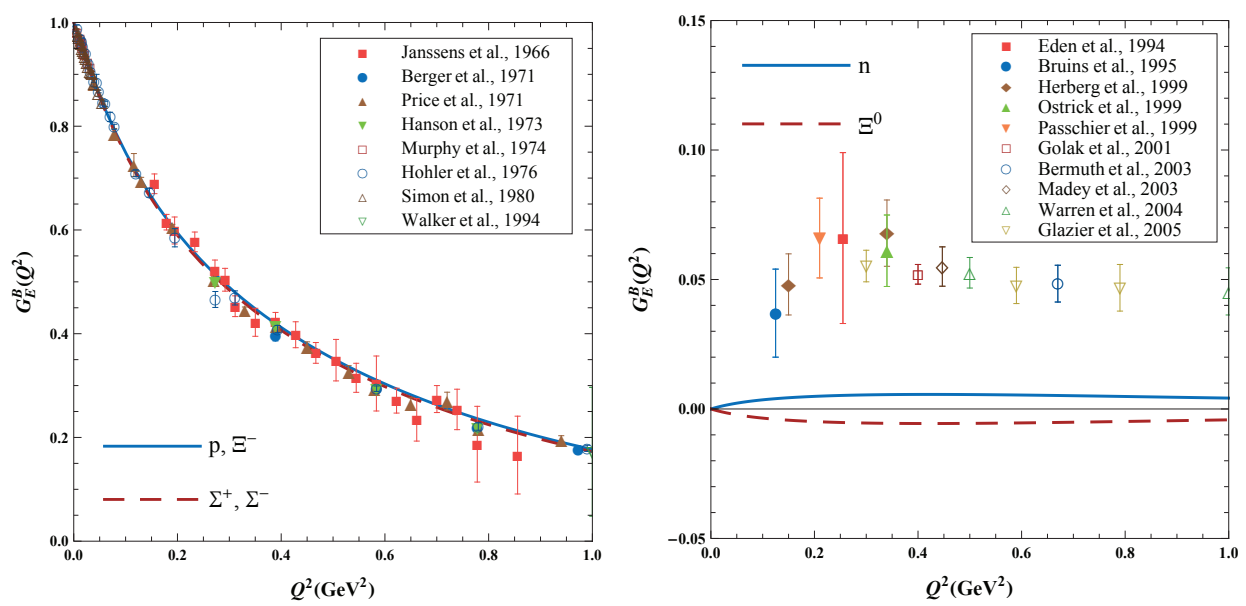

Fig. 3. Charge form factors $G_{E}^{B}\left(Q^{2}\right)$ of octet baryons. The experimental data on $G_{E}^{p}\left(Q^{2}\right)$ taken from Refs. 13-20, and $G_{E}^{n}\left(Q^{2}\right)$ taken from Refs. 21-30.

In Fig. 3, we present the $Q^{2}$ dependence of the charge form factor of the charged and neutral baryons respectively in the region $Q^{2} \leq 1 \mathrm{GeV}^{2}$, compared with the experimental data. ${ }^{13-30}$ It is seen in the left panel of Fig. 3 that the theoretical charge form factor of proton is well fitted to the experimental data, and the charge form factors for the charged hyperons $\left(\Sigma^{+}, \Sigma^{-}\right.$and $\left.\Xi^{-}\right)$derived with the predetermined wave function behave the similar way since our calculations are restricted to the $\mathrm{SU}(3)$ chiral symmetry. However, the neutron form factor is rather smaller than the experimental data, as shown in the right panel of Fig. 3, while the contributions to the charge form factor of $\Sigma^{0}$ and $\Lambda$ by various diagrams counteract each other to zero. The reason might be that the quark propagator is restricted to the ground-state only in our calculation. The meson cloud solely contributes to the neutral baryon charge form factors as the leading-order contribution of the $3 \mathrm{q}$-core vanishes. One may propose that it is necessary to include excited-state quarks to investigate the neutral baryon charge form factors. More discussions and results on the neutron charge radius including the excited quark propagator may be found in Refs. 3 and 4.

The fact that the $Q^{2}$ dependence of the theoretical charge form factor in the region $Q^{2} \leq 1 \mathrm{GeV}^{2}$ is consistent with experimental data implies that the predetermined quark wave function is reasonable in the PCQM. We expect that the predetermined quark wave function is applicable to other form factors of baryon octet. 


\section{Acknowledgments}

This work is supported by Suranaree University of Technology (SUT). XL and KK acknowledge support from SUT scholarship and TRF-CHE (MRG5080374), respectively.

\section{References}

1. V. E. Lyubovitskij, Th. Gutsche, A. Faessler and E. G. Drukarev Phys. Rev. D 63, 054026 (2001).

2. V. E. Lyubovitskij, Th. Gutsche, A. Faessler, and M. R. Vinh Phys. Rev. C 65, 025202 (2002).

3. V. E. Lyubovitskij, Th. Gutsche and A. Faessler Phys. Rev. C 64, 065203 (2001).

4. S. Cheedket, V. E. Lyubovitskij, Th. Gutsche, A. Faessler, K. Pumsa-ard and Y. Yan Eur. Phys. J. A 20, 317 (2004).

5. K. Khosonthongkee, V. E. Lyubovitskij, Th. Gutsche, A. Faessler, K. Pumsa-ard, S Cheedket and Y Yan J. Phys. G: Nucl. Part. Phys. 32, 793 (2004).

6. A. Faessler, Th. Gutsche, V. E. Lyubovitskij and C. Oonariya J. Phys. G: Nucl. Part. Phys. 35, 025005 (2008).

7. V. E. Lyubovitskij, Th. Gutsche, A. Faessler and M. R. Vinh Phys. Lett. B 520, 204 (2001).

8. K. Pumsa-ard, V. E. Lyubovitskij, Th. Gutsche, A. Faessler and S. Cheedket Phys. Rev. C 68, 015205 (2003).

9. Y. Dong, A. Faessler, Th. Gutsche, J. Kuckei, V. E. Lyubovitskij, K. Pumsa-ard and P. Shen J. Phys. G: Nucl. Part. Phys. 32, 203 (2006).

10. C. Dib, A. Faessler, Th. Gutsche, S. Kovalenko, J. Kuckei, V. E. Lyubovitskij and K. Pumsa-ard J. Phys. G: Nucl. Part. Phys. 32, 547 (2006).

11. G. Murray and L. Francis Phys. Rev. 84, 350 (1951).

12. M. Rotenberg Adv. At. Mol. Phys. 6, 233 (1970).

13. T. Janssens, R. Hofstadter, E. B. Hughes and M. R. Yearian Phys. Rev. 142, 922 (1966).

14. C. Berger, V. Burkert, G. Knop, B. Langenbeck and K. Rith Phys. Lett. B 35, 87 (1971).

15. L. E. Price, J. R. Dunning, M. Goitein, K. Hanson, T. Kirk and R. Wilson Phys. Rev. D 4, 45 (1971).

16. K. M. Hanson, J. R. Dunning, M. Goitein, T. Kirk, L. E. Price and R. Wilson Phys. Rev. D 8, 753 (1971).

17. J. J. Murphy, Y. M. Shin and D. M. Skopik Phys. Rev. C 9, 2125 (1971).

18. G. Höhler, E. Pietarinen, I. Sabba-Stefanescu, F. Borkowski, G. G. Simon, V. H. Walther and R. D. Wendling Nucl. Phys. B 114, 505 (1976).

19. G. G. Simon, C. Schmitt, F. Borkowski and V. H. Walther Nucl. Phys. A 333, 381 (1980).

20. R. C. Walker et al Phys. Rev. D 49, 5671 (1994).

21. T. Eden et al Phys. Rev. C 50, R1749 (1994)

22. E. E. W. Bruins et al Phys. Rev. Lett. 75, 21 (1995).

23. C. Herberg et al Eur. Phys. J. A 5, 131 (1999).

24. M. Ostrick et al Phys. Rev. Lett. 83, 276 (1999).

25. I. Passchier et al Phys. Rev. Lett. 82, 4988 (1999). 
26. J. Golak, G. Ziemer, H. Kamada, H. Witała and W. Glöckle 2001 Phys. Rev. C 63, 034006

27. J. Bermuth et al Phys. Lett. B 564, 199 (2003).

28. R. Madey et al Phys. Rev. Lett. 91, 122002 (2003).

29. G. Warren et al Phys. Rev. Lett. 92, 042301 (2004).

30. D. I. Glazier et al Eur. Phys. J. A 24, 101 (2005) 